\title{
Standard Model Predictions for High Mass Photon Pairs in $\ell \ell$ Events at LEP
}

M. Martinez and R. Miquel

CERN, CH-1211 Geneva 23, Switzerland

\begin{abstract}
Standard Model predictions for the processes $e^{+} e^{-} \rightarrow \ell \bar{\ell} \gamma \gamma$ with $\ell=\nu, e, \mu$ and $\tau$ are computed using a complete matrix element calculation keeping all masses. Numerical results are given for the region with high two-photon invariant mass.
\end{abstract}




\section{Introduction}

The high statistics accumulated by the LEP experiments allows already detailed studies of events with low probability, searching for small signals from new physics [1,2]. These studies will become even more important in the future, given the expected LEP high luminosity. Therefore, it is important that a reliable estimation of the Standard Model predictions exists.

Recently there has been some interest in the Standard Model prediction for the process $e^{+} e^{-} \rightarrow \ell \bar{\ell} \gamma \gamma$ to test the agreement with the data shown in [2]. In there the data is compared to the prediction in [3], which does not include the complete matrix element up to $\mathcal{O}\left(\alpha^{2}\right)$. Comparisons with [4,5] are also mentioned. In both cases no masses are included and in [5] there is no initial state radiation. Furthermore, no comparion is possible for the process $e^{+} e^{-} \rightarrow e^{+} e^{-} \gamma \gamma$, since the prediction is not included in any of the above references [3]-[5].

In view of this situation, we have undertaken the task of putting together a complete $\mathcal{O}\left(\alpha^{2}\right)$ calculation of the process $e^{+} e^{-} \rightarrow \ell \bar{\ell} \gamma \gamma$ valid for all leptons $(\ell=e, \mu, \tau, \nu)$, including photon and $Z$ exchange, initial and final state radiation and keeping all masses.

\section{Calculation}

Given the fact that for $e^{+} e^{-} \rightarrow e^{+} e^{-} \gamma \gamma$ there are as many as 80 Feynman diagrams which contribute, the most efficient way to face the problem is the numerical implementation of the helicity amplitudes technique [6]-[11]. Following the aim of producing a calculation as general as possible, out of the differents approaches existing in the literature we have chosen to use one in which masses are rigorously kept [12]. For the configuration in which the angles among all the particles in the process are sizable, keeping the masses in the calculation is not relevant (except, maybe for the $\tau^{+} \tau^{-} \gamma \gamma$ channel) but, if one of these angles is allowed to be small, then masses cannot be neglected. Since this configuration can become also interesting in the future, we would like to keep the generality in our matrix element squared to enable its use in any region of the 4 -body phase space.

The calculation for $e^{+} e^{-} \rightarrow e^{+} e^{-} \gamma \gamma$ when only photon t-channel diagrams are considered is described in detail in ref. [13] and therefore we will not repeat it here. Following the receipts of ref. [12], the inclusion of s-channel diagrams and of $\mathrm{Z}$ exchange is straightforward. Once the calculation of $e^{+} e^{-} \rightarrow e^{+} e^{-} \gamma \gamma$ is complete, the ones for $e^{+} e^{-} \rightarrow \mu^{+} \mu^{-} \gamma \gamma$ and $e^{+} e^{-} \rightarrow \tau^{+} \tau^{-} \gamma \gamma$ are obtained by just dropping the t-channel contributions and setting the corresponding final state particle masses.

The matrix element squared for the process $e^{+} e^{-} \rightarrow \nu \bar{\nu} \gamma \gamma$ can be found in ref. [14]. It can be obtained from the $e^{+} e^{-} \rightarrow \mu^{+} \mu^{-} \gamma \gamma$ matrix element squared by just dropping the photon contribution and setting the couplings and masses properly. By doing so, only the s-channel Z-exchange diagrams are accounted for but, since they constitute a gauge-invariant set and the contribution of the missing diagrams is very small [14], they can be safely neglected.

Our matrix element squared can be used as well for quark production, by just setting properly the couplings and masses. Nevertheless, in this case, the proper inclusion of QCD corrections and fragmentation is mandatory. Since this work goes beyond the scope of our present analysis, we will not discuss it further.

Some checks of both matrix elements squared (for charged and neutral leptons, respectively) have been performed such as testing gauge invariance by changing the photon polarization vector 
or comparing partial results with results obtained using massless trace reduction techniques.

For the actual integration of the matrix element squared, the choice of the phase-space point distribution is directly connected to the actual phase-space region of interest. Since in the numerical analysis we will concentrate in the predictions for high mass photon pairs following the cuts of reference [2] we have decided to use the flat phase-space generator RAMBO [15]. The reason is twofold:

- first, the detection cuts avoid reasonably the QED peaks due to collinear configurations.

- the flat phase-space generation tends to put more points in the high photon invariant mass region, in which we are mostly interested.

To check that no peak has been overseen in the integration, we have tried to use a phase space generator allowing the control over the variables which produce the matrix element squared dominant behaviour:

- for the charged lepton channels, since final state radiation clearly dominates, a phase-space parametrization representing the decay of a pair of virtual leptons into a real lepton and a photon each, has been used:

$$
\begin{aligned}
d^{8} R_{4}= & d^{2} R_{2}\left(s ; s_{+}, s_{-}\right) d^{2} R_{2}\left(s_{+} ; m_{f}^{2}, 0\right) d^{2} R_{2}\left(s_{-} ; m_{f}^{2}, 0\right) d s_{+} d s_{-} \quad \text { being } \\
d^{2} R_{2}\left(s ; s_{+}, s_{-}\right) & =\frac{1}{8}\left(1-\frac{s_{+}+s_{-}}{s}\right)\left(1-\frac{4 s_{+} s_{-}}{\left(s-s_{+}-s_{-}\right)^{2}}\right)^{\frac{1}{2}} d \cos \theta d \phi \\
d^{2} R_{2}\left(s^{\prime} ; m_{f}^{2}, 0\right)= & \frac{1}{8}\left(1-\frac{m_{f}^{2}}{s^{\prime}}\right) d \cos \theta_{f}^{*} d \phi_{f}^{*}
\end{aligned}
$$

where $s^{\prime}$ stands for $s_{+}$or $s_{-}$and the $*$ superindex denotes the virtual lepton rest frame.

- for the neutrino channel, a phase-space parametrization representing the production of two photons and a virtual $\mathrm{Z}$ decaying into two neutrinos has been used:

$$
\begin{aligned}
d^{8} R_{4}= & d^{5} R_{3}\left(s ; s^{\prime}, 0,0\right) d^{2} R_{2}\left(s^{\prime} ; 0,0\right) d s^{\prime} \quad \text { being } \\
& d^{5} R_{3}\left(s ; s^{\prime}, 0,0\right)=\frac{s}{512} \frac{x_{1} x_{2}^{2}}{1-x_{1}-s^{\prime} / s} d x_{1} d \cos \theta_{1} d \phi_{1} d \cos \theta_{2} d \phi_{2} \\
& d^{2} R_{2}\left(s^{\prime} ; 0,0\right)=\frac{1}{8} d \cos \theta_{\nu}^{*} d \phi_{\nu}^{*}
\end{aligned}
$$

where the index $i=1,2$ labels the two photons, $x_{i} \equiv E_{\gamma i} / E_{\text {beam }}$ and the $*$ superindex denotes the virtual $\mathrm{Z}$ rest frame.

In both cases, the adaptive Monte Carlo integration program VEGAS [16] has been used to map the cross section peaks into the integration variables and produce a reliable estimate of the 
integral. In the phase-space region of interest, the agreement between the RAMBO approach and the most elaborated one just described is excellent.

The calculation described so far follows a pure Born approach. Nevertheless, the effect of higher orders can be accounted for with a few percent accuracy (which is our goal) by using the following scheme:

- the bulk of the non-photonic corrections can be introduced by simply identifying the Born couplings with the measured values of the "improved Born" effective parameters.

- the bulk of the soft and collinear initial state photon emision corrections, can be accounted for by just multiplying the "improved Born" cross section by a cutoff radiation exponentiated factor:

$$
\sigma \simeq \sigma_{\text {Born }}\left(\frac{\Gamma_{Z}}{M_{Z}}\right)^{\beta}\left(1+\frac{3}{4} \beta\right) \simeq 0.75 \sigma_{\text {Born }}
$$

being

$$
\beta=\frac{2 \alpha}{\pi}\left(\ln \frac{s}{m_{e}^{2}}-1\right)
$$

For the charged leptons final state, since final state radiation dominates by far, the initial state radiation correction can be introduced with more accuracy by using directly the ratio between the experimental values of the measured cross section at the peak energy and the deconvoluted peak cross section. Therefore, we have chosen not to correct for this effect the numbers given in the following section.

\section{Results}

Figure 1 shows our prediction for the differential cross section with respect to the photon invariant mass for the four lepton species within the kinematical cuts used in ref. [2] (see Table 1 for details). As can be seen, the shape of the distribution for the charge leptons is basically the same whereas their normalisation is different (mainly due to the different detection cuts applied to each one). For the neutrinos, the shape and the normalization are completely different due to the fact that, in that case, photons come only from initial state radiation.

In Table 1 we give the integrated cross section for photon invariant masses $M_{\gamma \gamma}$ larger than 20 and $50 \mathrm{GeV}$, respectively. The errors quoted come from the limited Monte Carlo integration statistics used.

\begin{tabular}{|c||c|c|c|c|c|}
\hline Process & $E_{\ell}(\mathrm{GeV})$ & $\left|\cos \theta_{\ell}\right|$ & $\theta_{\ell \gamma}$ & $\sigma(p b)\left[M_{\gamma \gamma}>20 \mathrm{GeV}\right]$ & $\sigma(p b)\left[M_{\gamma \gamma}>50 \mathrm{GeV}\right]$ \\
\hline \hline$e^{+} e^{-} \gamma \gamma$ & $>3$ & $<0.74$ & $>8^{\circ}$ & $0.426 \pm 0.016$ & $0.0324 \pm 0.0011$ \\
\hline$\mu^{+} \mu^{-} \gamma \gamma$ & $>3$ & $<0.8$ & $>5^{\circ}$ & $0.516 \pm 0.016$ & $0.0385 \pm 0.0014$ \\
\hline$\tau^{+} \tau^{-} \gamma \gamma$ & $>3$ & $<0.74$ & $>15^{\circ}$ & $0.168 \pm 0.003$ & $0.0105 \pm 0.0003$ \\
\hline \hline$\nu \bar{\nu} \gamma \gamma$ & nil & nil & nil & $(0.397 \pm 0.013) \times 10^{-3}$ & $(1.86 \pm 0.02) \times 10^{-6}$ \\
\hline
\end{tabular}

Table 1: Integrated improved Born cross section for $\sqrt{s}=91.2 \mathrm{GeV}$ for the different processes contributing to the $\ell \bar{\ell} \gamma \gamma$ final state. For all channels $E_{\gamma}>1 \mathrm{GeV}$ and $\left|\cos \theta_{\gamma}\right|<0.9$ is also required. The electroweak parameters used are: $M_{Z}=91.187 \mathrm{GeV}, \Gamma_{Z}=2.492 \mathrm{GeV}$ and $\sin ^{2} \theta_{\text {eff }}=0.2324$. 
We see from the table that in the very high mass region $\left(M_{\gamma \gamma}>50 \mathrm{GeV}\right)$, the combined cross section for the three charged channels amounts to about $0.08 \mathrm{pb}$ while the similar result in the neutrino channel is down by more than four orders of magnitude, as expected from the fact that there is no final state radiation in this latter process.

\section{Conclusions}

A complete matrix element squared for the process $e^{+} e^{-} \rightarrow \ell \bar{\ell} \gamma \gamma$ has been computed in order to evaluate the Standard Model prediction for the observation of high mass photon pairs in leptonic events at LEP. The fact that masses have been rigorously kept allows the study of any phase space region. The generality of the calculation also allows an easy implementation of the electroweak prediction for quark final states.

Numerical results relevant for the high photon invariant mass region have been presented. Inside the kinematical cuts of ref. [2] the cross section for photon pair masses over $50 \mathrm{GeV}$ is found to be around $0.08 \mathrm{pb}$ combining the electron, muon and tau channel. Much smaller yields are found coming from the neutrino channel.

The FORTRAN code of the calculation can be obtained from MARTINEZ@CERNVM or MIQUEL@CERNVM.

After this work was completed we became aware of ref. [17] in which a similar calculation keeping all masses is presented for the $\mu^{+} \mu^{-} \gamma \gamma$ and $\tau^{+} \tau^{-} \gamma \gamma$ channels.

\section{Acknowledgements}

We thank G. Bonvicini for attracting our attention towards this subject. We also thank J. Lefrançois and D. Schlatter for their interest. We are indebted to J. Knobloch for his help in computing aspects. 


\section{References}

[1] D. Decamp et al. (ALEPH Coll.), Z. Phys. C52 (1992) 1.

[2] The L3 Collaboration, CERN-PPE/92-152, September 1992. To appear in Phys. Lett. B.

[3] S. Jadach and B.F.L.Ward, Phys. Lett. B274 (1992) 470.

[4] S. Jadach et al., Phys. Rev. D42 (1990) 2977.

[5] W. J. Stirling, Phys. Lett. B271 (1991) 261.

[6] CALKUL Coll.:

P. de Causmacker et al., Nucl. Phys. B206 (1982) 53.

F. A. Berends et al., Nucl. Phys. B264 (1986) 243 and 265.

[7] G. R. Ferrar and F. Nevi, Phys. Lett. B130 (1983) 109.

[8] S. Jadach and Z. Wąs, Acta Phys. Pol. B15 (1984) 1151.

[9] F. A. Berends, P. H. Daverveldt and R. Kleiss, Nucl. Phys. B253 (1985) 441.

[10] R. Kleiss and W. J. Stirling, Nucl. Phys. B262 (1985) 235.

[11] C. Mana and M. Martinez, Nucl. Phys. B287 (1987) 601.

[12] M. Martinez, C. Mana and R. Miquel, "Helicity Amplitudes Calculation", Proceedings of the Workshop on QED structure functions, Ann Arbor, Michigan, USA. American Institute of Physics, Particles and Fields series Vol.39 (1989) 243 and references therein.

[13] C. Mana, M. Martinez and R. Miquel,"Radiative corrections to the neutrino counting experiment", Proceedings of the Workshop on QED structure functions, Ann Arbor, Michigan, USA. American Institute of Physics, Particles and Fields series Vol.39 (1989) 395.

R. Miquel, Ph. D. thesis. UAB-LFAE 89-02 (1989).

M. Martinez, R. Miquel, Universitat Autònoma de Barcelona preprint, UAB-LFAE 87-01 (1987), unpublished.

[14] F. A. Berends et al. Nucl. Phys. B301 (1988) 583.

R. Miquel, M. Martinez and C. Mana, Z. Phys. C48 (1990) 309.

[15] R. Kleiss and W.J. Stirling, Comp. Phys. Comm. 40 (1986) 359.

[16] G. P. Lepage, J. Comp. Phys. 27 (1978) 192.

[17] D. J. Summers, Durham University preprint, DTP/92/76, October 1992 


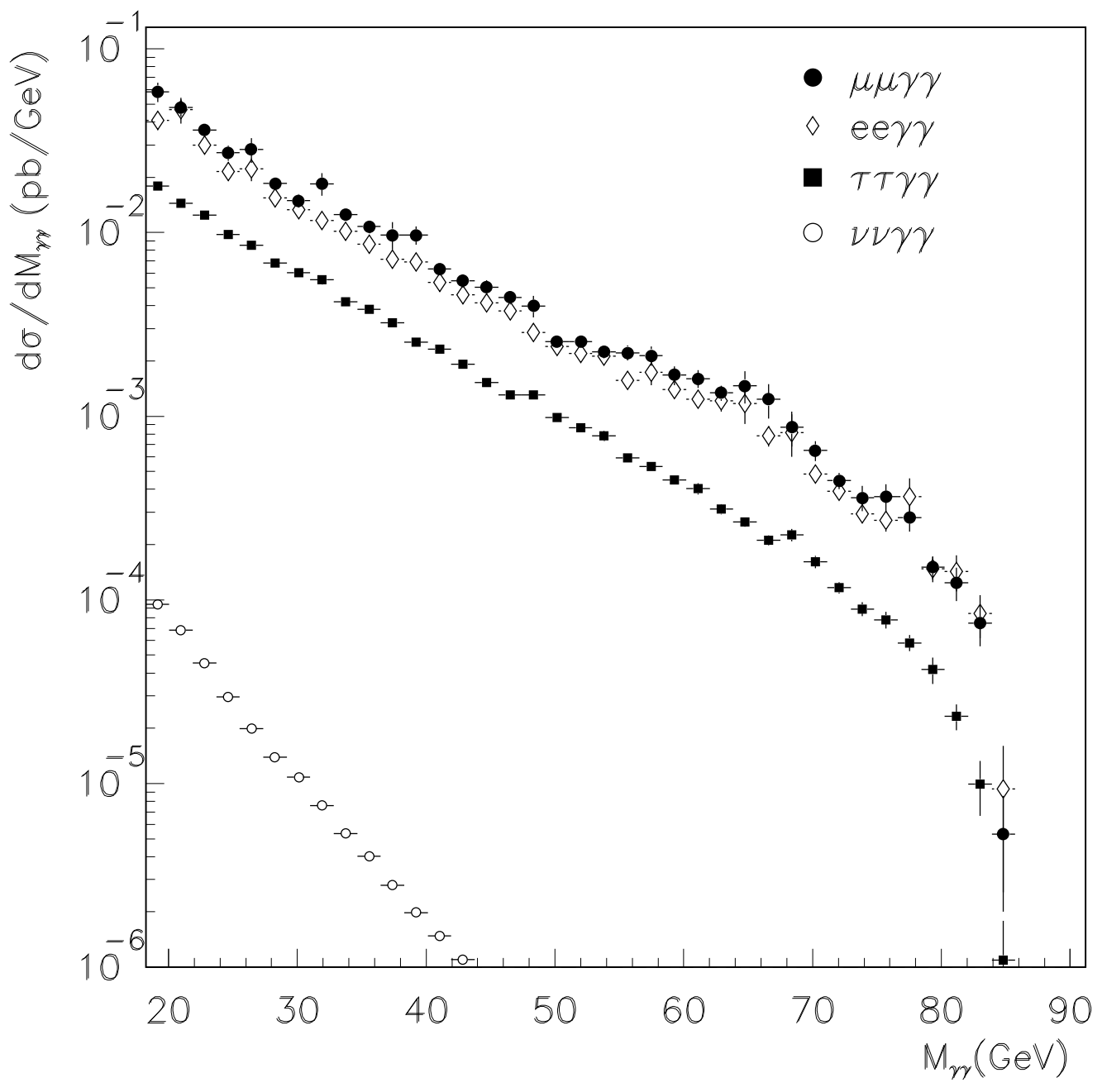

Figure 1: Photon invariant mass differential cross sections at $\sqrt{s}=91.2 \mathrm{GeV}$. The kinematical cuts and the values of the electroweak parameters used are defined in Table 1 . The errors shown come from Monte Carlo statistics. 